\title{
Long non-coding RNA UCA1 confers tamoxifen resistance in breast cancer endocrinotherapy through regulation of the EZH2/p21 axis and the PI3K/AKT signaling pathway
}

\author{
ZHUO LI ${ }^{1}$, DEHAI YU ${ }^{2}$, HAIJUN LI ${ }^{3}$, YOU LV ${ }^{1}$ and SIJIE LI ${ }^{4}$ \\ ${ }^{1}$ Endocrinology and Metabolism Department, The First Hospital of Jilin University; ${ }^{2}$ Cancer Center, \\ The First Hospital of Jilin University; ${ }^{3}$ Institute of Translational Medicine, The First Hospital of Jilin University; \\ ${ }^{4}$ Department of Breast Surgery, The First Hospital of Jilin University, Changchun, Jilin 130021, P.R. China
}

Received July 9, 2018; Accepted October 12, 2018

DOI: 10.3892/ijo.2019.4679

\begin{abstract}
Tamoxifen is the gold standard for breast cancer endocrinotherapy. However, drug resistance remains a major limiting factor of tamoxifen treatment. Long non-coding (lnc) RNA serves an important role in drug resistance; however, the molecular mechanisms of tamoxifen resistance in breast cancer endocrinotherapy are largely unclear. IncRNA urothelial cancer associated 1 (IncRNA UCA1, UCA1) has been proven to be dysregulated in human breast cancer and promotes cancer progression. In the present study, it was demonstrated that UCA1 was significantly upregulated in breast cancer tissues compared with healthy tissues. Furthermore, the expression level of UCA1 was significantly greater in tamoxifen-resistant breast cancer cells (LCC2 and LCC9) when compared with those in the tamoxifen-sensitive breast cancer cells (MCF-7 and T47D). UCA1 silencing in LLC2 and LLC9 cells increased tamoxifen drug sensitivity by promoting cell apoptosis and arresting the cell cycle at the $\mathrm{G}_{2} / \mathrm{M}$ phase. Notably, the induced overexpression of UCA1 in MCF-7 and T47D cells decreased the drug sensitivity of tamoxifen. The molecular mechanism involved in UCA1-induced tamoxifen-resistance was also investigated. It was identified that UCA1 was physically associated with the enhancer of zeste homolog 2 (EZH2), which suppressed the expression of p21 through histone methylation (H3K27me3) on the 21 promoter. In addition, it was demonstrated that UCA1 expression was paralleled to the phosphorylation of CAMP responsive element binding protein (CREB) and AKT. When LCC2 cells were treated with the phosphoinositide 3-kinase (PI3K)/protein kinase B (AKT) signaling pathway
\end{abstract}

Correspondence to: Professor Sijie Li, Department of Breast Surgery, The First Hospital of Jilin University, Xinmin Street, Changchun, Jilin 130021, P.R. China

E-mail: lisijie412@126.com

Key words: long non-coding RNA, urothelial cancer associated 1, breast cancer, endocrinotherapy, drug resistance inhibitor LY294002, the phosphorylation levels of CREB and AKT were significantly downregulated. Taken together, it was concluded that UCA1 regulates the EZH2/p21 axis and the PI3K/AKT signaling pathway in breast cancer, and may be a potential therapeutic target for solving tamoxifen resistance.

\section{Introduction}

Breast cancer is the most common female malignancy and the second most common cause of cancer-associated fatality in the world (1). Approximately $70 \%$ of patients with breast cancer are estrogen receptor-positive (ER ${ }^{+}$(2). Apart from surgery, endocrine therapy (including tamoxifen, fulvestrant and letrozole) has improved the overall survival and quality of life for patients with breast cancer (2-4). Among all endocrine therapies, tamoxifen is the most extensively used hormone therapy and functions as an estrogen antagonist in breast cancer $(5,6)$. Although the majority of patients with ER+ breast cancer benefit from tamoxifen therapy, many tumors eventually recur because of tamoxifen resistance $(7,8)$. Tamoxifen resistance can arise via several mechanisms, including loss of $\mathrm{ER} \alpha$, induction of abnormal estradiol levels and alterations of coregulatory proteins, including amplified in breast cancer 1 and histone deacetylase (9-11).

An increasing number of long non-coding (lnc)RNAs in the human genome have been identified, and have provided new directions in cancer research (12). IncRNA, a class of non-protein coding transcripts with $>200$ nucleotides, regulates protein-coding genes during transcription and post-transcription in a sequence-specific manner (13-15). Importantly, lncRNAs in cancer cells are associated with the formation of tamoxifen resistance (16-18). However, only a few IncRNAs have been proposed to be clinically relevant biomarkers for tamoxifen resistance, such as H19 and homeobox antisense intergenic RNA (19-21). Searching for appropriate lncRNAs is valuable for the management of tamoxifen-resistance.

Out of the numerous cancer-associated lncRNAs, IncRNA urothelial carcinoma-associated 1 (UCA1) serves an important oncogenic role in several cancer types, including bladder cancer, colorectal cancer and gastric cancer (22). UCA1 has three exons that encode a 1.4-kb isoform and a 2.2-kb isoform (23). It was originally identified as a urine marker (the $1.4-\mathrm{kb}$ isoform) in 
bladder cancer (24). Tuo et al (25) demonstrated that UCA1 can modulate breast cancer cell growth and apoptosis through downregulation of the tumor suppressor microRNA (miR)-143. Huang et al (23) reported that UCA1 can promote breast tumor growth by suppressing the level of p27. UCA1 is also associated with the poor prognosis of cancer. Bian et al (26) demonstrated that patients with colorectal cancer and higher UCA1 expression had a significantly poorer prognosis. Furthermore, it was reported that UCA1 expression was correlated with a reduction in recurrence-free survival in breast cancer (27). These findings highlight the important role of UCA1 in cancer development.

The polycomb group protein enhancer of zeste homolog 2 (EZH2) is a critical regulator of tumorigenesis $(28,29)$. It has been demonstrated that the level of EZH2 is elevated in human bladder cancer, breast cancer, colon cancer and prostate cancer (30). Furthermore, the expression and mutation of EZH2 can regulate the level of $\mathrm{H} 3 \mathrm{~K} 27 \mathrm{me} 3$ (31). In hepatocellular carcinoma, UCA1 repressed p27 expression through its association with EZH2, which suppresses p27Kip1 through H3K27me3 on the p27Kip1 promoter (32). However, the effects of UCA1 on EZH2 expression and the underlying molecular mechanisms in breast cancer are not fully understood.

The phosphoinositide 3-kinase (PI3K)/protein kinase B (AKT) signaling pathway is the most frequently altered pathway in human cancer, and previous studies have demonstrated that UCA1 regulates the cell cycle progression of bladder carcinoma cells via PI3K/AKT-dependent signaling $(33,34)$. Notably, the knockdown of UCA1 inhibits AKT phosphorylation in breast cancer cells (35). Additionally, activation of the PI3K/AKT signaling pathway has been demonstrated to confer resistance to antiestrogens in tamoxifen-resistant breast cancer cells (36). Therefore, it would be useful to determine whether UCA1 is involved in the PI3K/AKT signaling pathway and if it induces tamoxifen resistance in breast cancer cells.

In the present study, the level of UCA1 expression was investigated in tamoxifen-resistant cells and compared with tamoxifen-sensitive cells. Induction of UCA1 overexpression in MCF-7 and T47D breast cancer cells and silencing of UCA1 in LLC2 and LLC9 breast cancer cells was performed to assess the drug sensitivity of the cells to tamoxifen. Furthermore, it was explored whether UCA1 was physically associated with $\mathrm{EZH} 2$. In addition, it was investigated whether UCA1 regulates tamoxifen resistance through a EZH2/p21 axis and the PI3K/AKT signaling pathway in breast cancer.

\section{Materials and methods}

Patients and specimens. A total of 10 hormone receptor-positive breast cancer specimens and 10 non-tumor specimens were randomly selected from the First Hospital of Jilin University (Changchun, China) between April 2015 and April 2017. All these participants were female. The breast cancer specimens were histologically diagnosed as breast carcinoma using ultrasound-guided core needle biopsy of the breast. In the 10 breast cancer specimens, 1 was at stage I, 5 were at stage II and 4 were at stage III. The age range of the 10 patients was from 37-68 years old, with a median age of 51. Evidence of bilateral disease and pregnancy concomitant with the diagnosis of breast cancer resulted in exclusion from the study. All samples were collected prior to tamoxifen therapy and stored in liquid nitrogen $\left(-196^{\circ} \mathrm{C}\right)$ until use. Permission to use the clinical samples for research purposes was obtained and approved by the Ethics Committee of the First Hospital of Jilin University. Informed consents were obtained from all patients.

Cell culture. Human breast cancer cell lines MCF-7 (tamoxifen-sensitive), T47D (tamoxifen-sensitive), LCC2 (tamoxifen-resistant) and LCC9 (tamoxifen-resistant), were purchased from American Type Culture Collection (Manassas, VA, USA). All cancer cells were maintained in Dulbecco's modified Eagle's medium (DMEM, Gibco; Thermo Fisher Scientific, Inc., Waltham, MA, USA) supplemented with $10 \%$ fetal bovine serum (FBS, HyClone, Logan, UT, USA) $2 \mathrm{mM}$ glutamine (Gibco; Thermo Fisher Scientific, Inc.), $100 \mathrm{U} / \mathrm{ml}$ penicillin and $100 \mu \mathrm{g} / \mathrm{ml}$ streptomycin (HyClone). Cells were cultured at $37^{\circ} \mathrm{C}$ in an incubator with a humidified atmosphere containing $5 \% \mathrm{CO}_{2}$.

Overexpression and knockdown of UCAl in breast cancer cells. To induce the overexpression of UCA1 in breast cancer cells, the cDNA encoding UCA1 was polymerase chain reaction (PCR)-amplified. The primer sequences were as follows: UCA1, forward 5'-CGCGGATCCTTTATCAGGCATATTAG CTTTAA-3' (BamHI) and reverse 5'-GCGAATTCTGACATTC TTCTGGACAATG-3' (EcoRI). Following this, the PCR product was subcloned into the pGreen.puro lentivirus vector (SBI, Palo Alto, CA, USA) with BamHI and EcoRI restriction sites (Takara Biotechnology Co. Ltd., Dalian, China). Viral particles were harvested at $48 \mathrm{~h}$ post-cotransfection of the pGreen-UCA1-puro constructs with the packaging plasmid ps-PAX2 and the envelope plasmid pMD2G (SBI) into 293T cells using Lipofectamine 3000 (Invitrogen; Thermo Fisher Scientific, Inc.). The empty vector was used as the control (lv-NC). MCF-7 and T47D cells were infected with the lentiviral particles $\left(5 \times 10^{7} \mathrm{TU} / \mathrm{ml}\right.$; lv-UCA1 or lv-NC) plus $6 \mu \mathrm{g} / \mathrm{ml}$ polybrene (Sigma-Aldrich; Merck KGaA, Darmstadt, Germany). The virus titers in the control and experimental groups were nearly the same as above $\left(\sim 5 \times 10^{7} \mathrm{TU} / \mathrm{ml}\right)$.

For the knockdown of UCA1, the small interfering (si)RNA targeting UCA1 (si-UCA1) and the scramble non-target control siRNA (si-NC) were synthesized by Shanghai GenePharma Co., Ltd., (Shanghai, China). si-UCA1 and si-NC sequences were as follows (37): si-UCA1, 5'-GTTAATCCAGGAGACAA AGA-3'; and si-NC, 5'-TTCTCCGAACGTGTCACGT-3'. LCC2 and LCC9 cells were transfected with equal amounts (100 nM) of si-UCA1 and si-NC using Lipofectamine 3000. All the following cellular or molecular experiments were carried out at $48 \mathrm{~h}$ post-transfection $(38,39)$.

RNA extraction, reverse transcription-PCR (RT-PCR) and $R T$-quantitative PCR (RT-qPCR). Total RNA was extracted from breast cancer tissues and cells using TRIzol reagent (Invitrogen; Thermo Fisher Scientific, Inc.) according to the manufacturer's instructions. cDNA was reverse transcribed using $1 \mu \mathrm{g}$ of total RNA and the SuperScript III First-Stand Synthesis Kit (Invitrogen; Thermo Fisher Scientific, Inc.). The expression level of UCA1 were determined on a PCR thermal cycler (T100, Bio-Rad Laboratories, Inc., Hercules, CA, USA) using $2 \mathrm{X}$ Taq PCR StarMix buffer (GeneStar, Beijing, China) 
or on a real-time PCR thermal cycler (ABI PRISM 7500, Applied Biosystems; Thermo Fisher Scientific, Inc.) using SYBR Green Master Mix (Applied Biosystems; Thermo Fisher Scientific, Inc.). The endogenous control gene was glyceraldehyde-3-phosphate dehydrogenase (GAPDH). The RT-PCR amplification process was as follows: 1 cycle at $98^{\circ} \mathrm{C}$ for $2 \mathrm{~min}$ and 32 cycles at $95^{\circ} \mathrm{C}$ for $20 \mathrm{sec}, 62^{\circ} \mathrm{C}$ for $15 \mathrm{sec}$, followed by $72^{\circ} \mathrm{C}$ for $15 \mathrm{sec}$; ending with an extension cycle at $72^{\circ} \mathrm{C}$ for $5 \mathrm{~min}$. The qPCR amplification process consisted of 1 cycle at $95^{\circ} \mathrm{C}$ for $10 \mathrm{~min}$, followed by 40 cycles at $95^{\circ} \mathrm{C}$ for $10 \mathrm{sec}$ and $58^{\circ} \mathrm{C}$ for $30 \mathrm{sec}$. The results of RT-PCR were visualized using a $3 \%$ agarose gel and qPCR was performed using the $2^{-\Delta \Delta \mathrm{Cq}}$ method. All the oligonucleotide primers were synthesized by Takara. The primer sequences used were as follows (33): UCA1, forward 5'-CTTCTGCATAGGATCTG CAATCAG-3' and reverse 5'-TTTTGTCCCCATTTTCCATCA TACG-3'; GAPDH, forward 5'-AGGTCGGAGTCAACGG ATTTG-3' and reverse 5'-GTGATGGCATGGACTGTGGT-3'.

WST-1 assay to assess cell viability. Tamoxifen was purchased from Sigma-Aldrich; Merck KGaA. The stock solution of tamoxifen $(500 \mu \mathrm{M})$ was prepared in $100 \% \mathrm{MeOH}$ and maintained at $4^{\circ} \mathrm{C}$. Working standard solutions at different concentrations were prepared by dilution in DMEM supplemented with $10 \%$ FBS. Solutions were added into the 96-well plate at a final concentration of $0,0.01,0.1,1,10$ or $100 \mu \mathrm{M}$ and incubated with cells for $24 \mathrm{~h}$. Following this, $10 \mu \mathrm{l}$ WST-1 (Roche Diagnostics, Shanghai, China) was added into each well and the cells were incubated at $37^{\circ} \mathrm{C}$ in the dark for $2 \mathrm{~h}$. The absorbances of 450 and $630 \mathrm{~nm}$ were monitored. The relative cell viability percentage in each group was calculated by comparison to that of the control group.

Flow cytometry for cell cycle and apoptosis analysis. LCC2 cells transfected with si-UCA1 (si-UCA1 LCC2) were treated with $10 \mu \mathrm{M}$ tamoxifen for $24 \mathrm{~h}$, trypsinized, collected and washed with PBS. For cell cycle analysis, cells were fixed in pre-cold $70 \%$ ethanol for $20 \mathrm{~min}$ and stored at $-20^{\circ} \mathrm{C}$. Following this, cells were washed with PBS and stained with a solution containing $3.5 \mu \mathrm{M}$ Tris- $\mathrm{HCl}$ (pH 7.6), $10 \mathrm{mM} \mathrm{NaCl}, 50 \mu \mathrm{g} / \mathrm{ml}$ propidium iodide (PI) (Sigma-Aldrich; Merck KGaA), $20 \mu \mathrm{g} / \mathrm{ml}$ RNase and 0.1\% igepal CA-630 (Sigma-Aldrich; Merck KGaA) for $20 \mathrm{~min}$ on ice to label DNA. Subsequently, LCC2 cells were analyzed using a FACSCaliber flow cytometer with a FlowJo software (version 10.0, BD Biosciences, Franklin Lakes, NJ, USA). The percentages of cells at different phases were calculated from three independent experiments.

For cell apoptosis analysis, cells were stained using an Annexin V-FITC/PI double staining apoptosis detection kit (BD Biosciences, San Jose, CA, USA) according to the manufacturer's instructions, and analyzed with a FACSCaliber flow cytometer. The cells in the different portions represented the different cell states as follows: The late-apoptotic cells were present in the upper right portion, the viable cells were present in the lower left portion and the early apoptotic cells were present in the lower right portion.

RNA immunoprecipitation (RIP) assay. The RIP experiment was performed in LCC2 cells using the Magna RIP RNA-Binding Protein Immunoprecipitation Kit
(EMD Millipore, Billerica, MA, USA) according to the manufacturer's instructions. EZH2 antibody for the RIP assay was purchased from Abcam (1:500; \#ab186006; Shanghai, China). Samples were treated with proteinase K (Thermo Fisher Scientific, Inc.) to digest the protein for $1 \mathrm{~h}$ at $37^{\circ} \mathrm{C}$ and the immunoprecipitated RNA was isolated. Final analysis of co-precipitated RNA was performed using qPCR and demonstrated as fold enrichment of UCA1.

Chromatin immunoprecipitation (ChIP) assay. The ChIP experiment was performed in LCC2 cells using the EZ ChIP Chromatin Immunoprecipitation Kit (\#17-371, EMD Millipore) according to the manufacturer's instructions. Briefly, LCC2 cells were incubated with formaldehyde for $10 \mathrm{~min}$ to generate DNA-protein cross-links; the crosslinked chromatin DNAs were sonicated into 200 to 1,000-bp-sized fragments. Subsequently, immunoprecipitation was performed using anti-EZH2 antibody (1:1,000; \#07-689, EMD Millipore) and anti-H3K27me3 antibody (1:1,000; \#17-622, EMD Millipore), or normal IgG (1:200, EZ ChIP Chromatin Immunoprecipitation Kit) as control. Precipitated chromatin DNA was recovered and analyzed by qPCR. The primer sequences of $\mathrm{p} 21$ promoter were as follows: Forward (40), 5'-AGACCATGTGGACCTGTCACTG-3' and reverse 5'-GTTTGGAGTGGTAGAAATCTGTC-3'.

Western blot analysis. Cell samples were lysed using radioimmunoprecipitation assay lysis buffer (Beyotime Institute of Biotechnology, Shanghai, China) containing protease inhibitor. The total protein concentration was determined using a BCA protein assay kit (Beyotime Institute of Biotechnology). A total of $20 \mu \mathrm{g}$ of total protein was loaded per lane and separated by SDS-PAGE (10 or $12 \%$ gels) and transferred to polyvinylidene fluoride membrane (Roche). The membranes were blocked in $5 \%$ skimmed milk diluted with Tris-buffered saline/Tween-20 (Tris-HCl 20 mmol/1, NaCl 150 mmol/l, 0.1\% Tween-20, pH 7.5) at room temperature for $1 \mathrm{~h}$ and subsequently incubated overnight at $4^{\circ} \mathrm{C}$ with primary antibodies: Anti-AKT (1:1,000, \#ab8805, Abcam), anti-phospho(p)-AKT (1:2,000, \#ab8933, Abcam), CAMP responsive element binding protein (CREB, 1:2,000, \#ab178322, Abcam), anti-p-CREB (1:1,000, \#ab10564, Abcam), anti-GAPDH (1:2,000, \#ab181603, AbMart Bio-tech Co. Ltd., Shanghai, China), anti-B cell lymphoma/leukemia-2 (Bcl-2, 1:2,000, \#ab196495, Abcam), anti-cleaved caspase-3 (1:1,000, \#9661, Cell Signaling Technology, Inc., Danvers, MA, USA), anti-cleaved caspase-9 (1:1,000, \#52873, Cell Signaling Technology, Inc.), anti-cyclin D1 (1:1,000,\#2978, Cell Signaling Technology, Inc.) and anti-p21 (1:1,000, \#2947, Cell Signaling Technology, Inc.). Subsequently, the membranes were incubated with anti-mouse (1:5,000, SAB3701214, Sigma-Aldrich; Merck KGaA) or rabbit (1:5,000, SAB3700852, Sigma-Aldrich; Merck $\mathrm{KGaA}$ ) horseradish peroxidase-conjugated secondary antibodies at $37^{\circ} \mathrm{C}$ for $1 \mathrm{~h}$. The immunoreactive bands were visualized using the ECL western blot substrate (Promega Corporation, Madison, WI, USA) and the relative band density was analyzed by Quantity-one software (version 4.6, Bio-Rad Laboratories, Inc.).

Suppression of the PI3K signaling pathway. The PI3K signaling pathway was suppressed by the PI3K inhibitor LY294002 (Cell Signaling Technology, Inc.). LCC2 cells were treated with $50 \mu \mathrm{M}$ LY294002 for $24 \mathrm{~h}$ in DMEM 

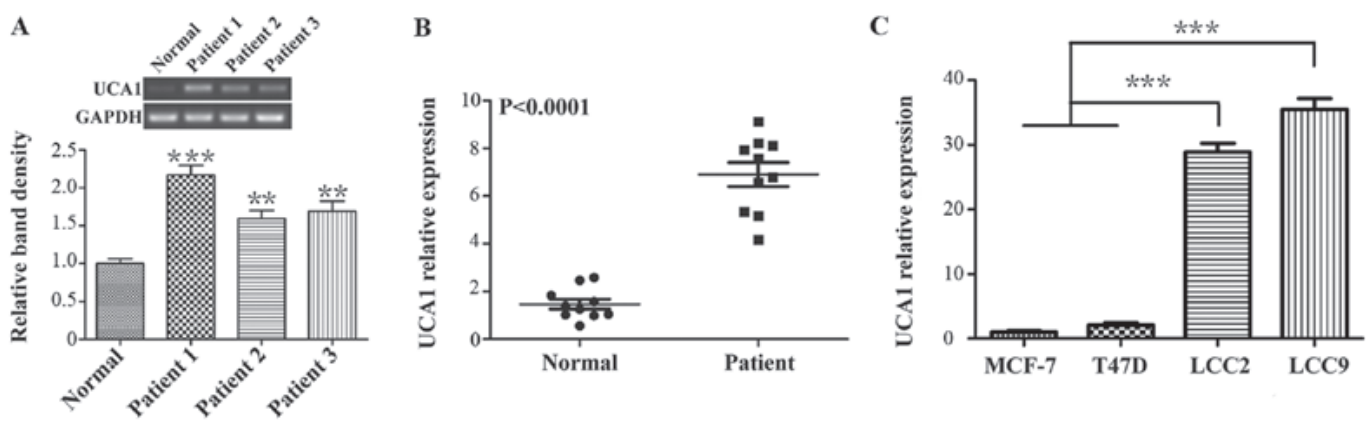

Figure 1. Expression level of UCA1 is higher in breast cancer. (A) Reverse transcription-polymerase chain reaction analysis of UCA1 expression in normal tissues and breast cancer tissues. (B) Quantitative polymerase chain reaction analysis of UCA1 expression in 10 normal and 10 breast cancer tissues. (C) Quantitative polymerase chain reaction analysis of UCA1 expression in tamoxifen-sensitive MCF-7 and T47D cells and in tamoxifen-resistant LCC2 and LCC9 cells. Data were analyzed using independent samples Student's t-test or one-way analysis of variance followed by a LSD or SNK test. ${ }^{* *} \mathrm{P}<0.01$ and ${ }^{* * *} \mathrm{P}<0.001$ as indicated or vs. normal group. UCA1, urothelial cancer associated 1.
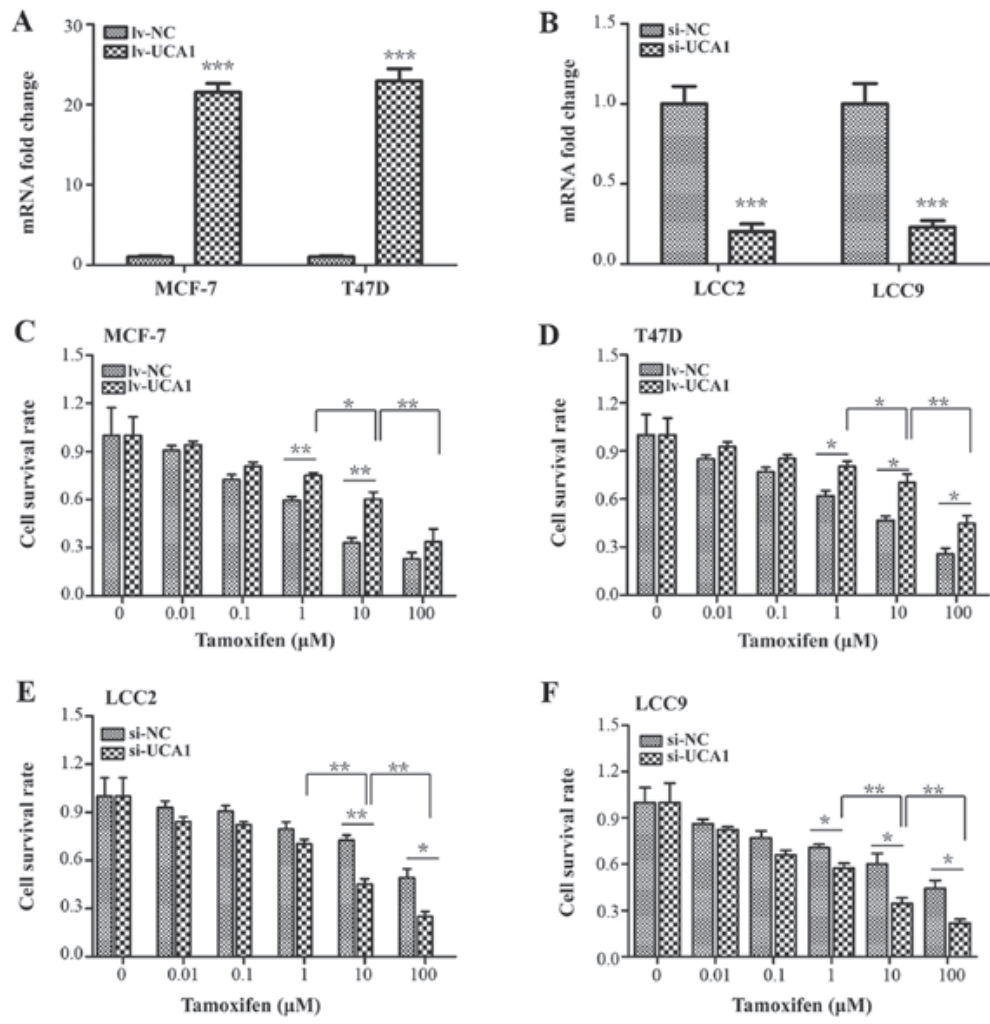

Figure 2. UCA1 contributes to tamoxifen resistance in breast cancer cells. Overexpression of UCA1 enhanced tamoxifen resistance in MCF-7 and T47D cells and knockdown of UCA1 enhanced tamoxifen sensitivity in LCC2 and LCC9 cells. (A and B) Delivery efficiency of the lentiviral carrying UCA1 DNA and the siRNA was assessed using quantitative polymerase chain reaction. (C and D) Overexpression of UCA1 increased the cell survival rate of MCF-7 and T47D cells. (E and F) Knockdown of UCA1 decreased the cell survival rate of LCC2 and LCC9 cells. Data were analyzed using the independent samples Student's t-test or one-way analysis of variance followed by a LSD or SNK test. ${ }^{*} \mathrm{P}<0.05,{ }^{* * *} \mathrm{P}<0.01$ and ${ }^{* * * *} \mathrm{P}<0.001$ as indicated or vs. control groups. UCA1, urothelial cancer associated 1; si, small interfering RNA; NC, negative control; lv, lentivirus.

supplemented with $10 \%$ FBS. Subsequent qPCR and western blot analysis were conducted at $24 \mathrm{~h}$ post-inhibition.

Statistical analysis. Data were presented as the mean \pm standard error of the mean of at least three independent experiments. Statistical significance between two groups was determined using one-way analysis of variance followed by an LSD or Dunnett's post hoc test or the Student's t-test. $\mathrm{P}<0.05$ was considered to indicate a statistically significant difference. All analyses were performed using SPSS 16.0 software (SPSS Inc., Chicago, IL, USA).

\section{Results}

UCA1 expression is upregulated in tamoxifen-resistant breast cancer cells. Firstly, 1 normal breast tissue and 3 breast cancer tissues were randomly selected from the 20 samples, and the level of UCA1 expression was detected using RT-PCR. The PCR results revealed that the level of UCA1 expression was significantly increased in breast cancer tissues compared with normal tissues $(\mathrm{P}<0.001$ and $\mathrm{P}<0.01$; Fig. $1 \mathrm{~A})$. Following this, the UCA1 expression levels in all the 10 normal breast tissues and 10 breast cancer tissues were assessed by qPCR. As indicated in 

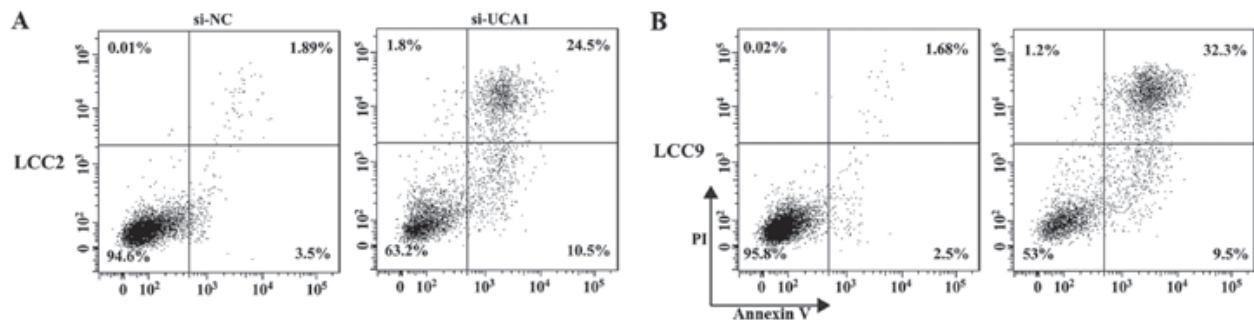

C

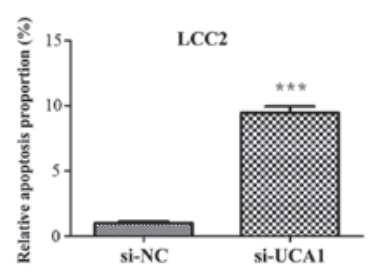

E
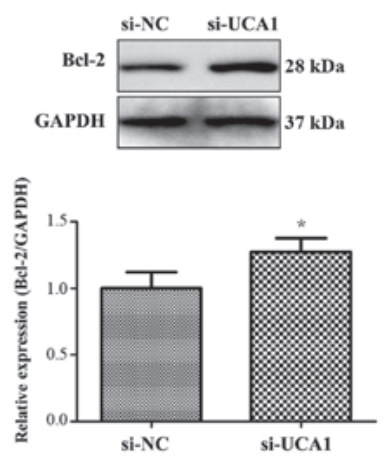

D

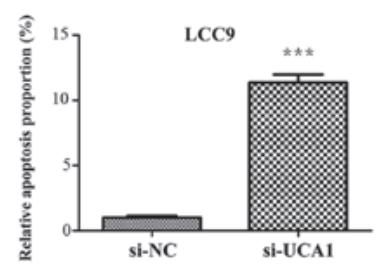

$\mathbf{F}$
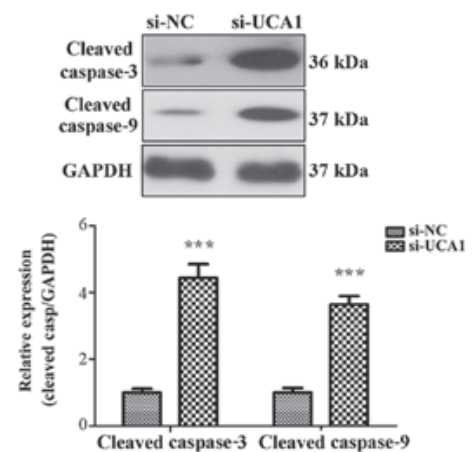

Figure 3. Knockdown of UCA1 increases cell apoptosis in tamoxifen-resistant breast cancer cells. (A and B) Flow cytometry analysis revealed cell apoptosis of LCC2 and LCC9 cells transfected with si-UCA1 increased following tamoxifen treatment. (C and D) Proportion of cell apoptosis in si-NC and si-UCA1 breast cancer cells following tamoxifen treatment. (E and F) Detection of cell apoptosis-associated factors, including Bcl-2, cleaved caspase-3 and -9 by western blot analysis. Upper panel, western blot bands; lower panel, relative band density to GAPDH. Data were analyzed using the independent samples Student's t-test. "P $<0.05$ and $^{* * * *} \mathrm{P}<0.001$ vs. control groups. UCA1, urothelial cancer associated 1; si, small interfering RNA; NC, negative control; Bcl-2, B-cell leukemia/lymphoma 2.

Fig. 1B, the mean expression level of UCA1 in the breast cancer group was 4.68 -fold greater when compared with that in the normal control group $(\mathrm{P}<0.0001)$. These data indicated a positive association between breast cancer and the expression of UCA1.

According to these results, the expression levels of UCA1 in tamoxifen-sensitive cells, MCF-7 and T47D, and in the tamoxifen-resistant cells, LCC2 and LCC9, were assessed using qPCR (Fig. 1C). It was revealed that the level of UCA1 expression in LCC2 and LCC9 cells was $>20$-fold greater when compared with that in MCF-7 and T47D cells $(\mathrm{P}<0.001)$, suggesting a positive association between tamoxifen resistance and UCA1 expression in breast cancer cells.

UCA1 affects the cell viability of breast cancer cells treated with tamoxifen. In order to further confirm the contribution of UCA1 to tamoxifen resistance, the WST-1 assay was performed to detect the cell survival rate following UCA1 knockdown or overexpression in breast cancer cells.

The delivery efficiencies of the lentivirus carrying UCA1 DNA and the siRNA were assessed. As indicated in Fig. 2A, UCA1 expression was significantly elevated by 21.67 - and 22.97-fold in lentivirus-transduced MCF-7 and T47D cells compared with the lv-NC group, respectively $(\mathrm{P}<0.001)$. Furthermore, UCA1 expression was significantly downregulated to 0.2 -fold and 0.23 -fold in the UCA1-siRNA transfected LCC2 and LCC9 cells when compared with the si-NC group, respectively (Fig. 2B; $\mathrm{P}<0.001$ ).
Following treatment with increasing concentrations of tamoxifen $(0,0.01,0.1,1,10$ and $100 \mu \mathrm{M})$, it was observed that the cell survival rates of UCA1-overexpressed cells were significantly increased compared with the lv-NC group in the presence of 1 or $10 \mu \mathrm{M}$ tamoxifen in MCF-7 cells and in the presence of 1,10 or $100 \mu \mathrm{M}$ tamoxifen in T47D cells (Fig. $2 \mathrm{C}$ and $\mathrm{D} ; \mathrm{P}<0.05$ and $\mathrm{P}<0.01$ ). Conversely, UCA1 silencing significantly decreased the cell survival rate compared with the si-NC group in the presence of 10 or $100 \mu \mathrm{M}$ tamoxifen in LCC 2 cells or in the presence of 1,10 or $100 \mu \mathrm{M}$ tamoxifen in LCC9 cells (Fig. 2E and F; P<0.05 and $\mathrm{P}<0.01$ ). Specifically, the cell survival rates significantly changed in the $10 \mu \mathrm{M}$ tamoxifen treatment group compared with the $1 \mu \mathrm{M}$ tamoxifen treatment group (Fig. 2C-F; P $<0.05$ or $\mathrm{P}<0.01)$. However, in the $100 \mu \mathrm{M}$ tamoxifen treatment group, the cell survival rates of the control and the experimental group were significantly decreased compared with the $10 \mu \mathrm{M}$ tamoxifen treatment group, indicating that a high concentration of tamoxifen promoted non-specific cytotoxicity (Fig. 2C-F; $\mathrm{P}<0.01)$.

Flow cytometry results indicated that the cell apoptosis rate of si-UCA1 LCC 2 cells (35\%) and si-UCA1 LCC9 cells $41.8 \%$ ) was significantly increased following $10 \mu \mathrm{M}$ tamoxifen treatment when compared with the negative control (si-NC, 5.39\% and 4.18\%; Fig. 3A-D; P<0.001). Several apoptosis-associated factors were also measured by western blot analysis. Results indicated that the expression levels of 
A

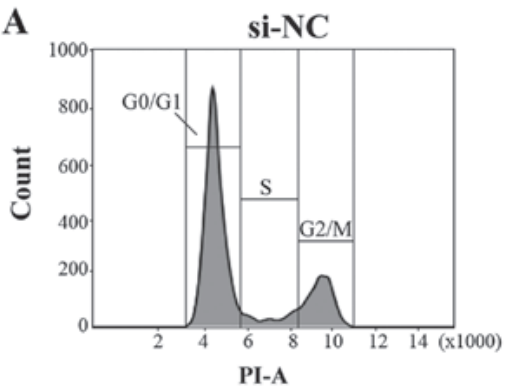

$\mathrm{C}$

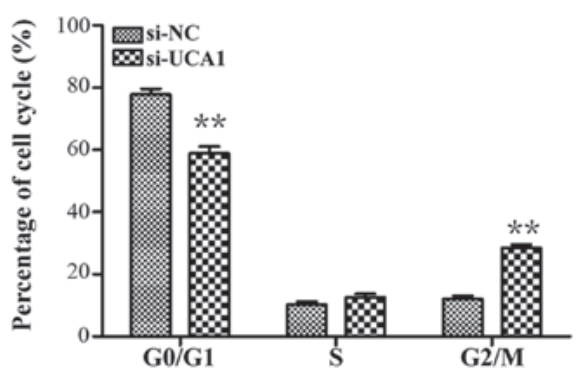

B

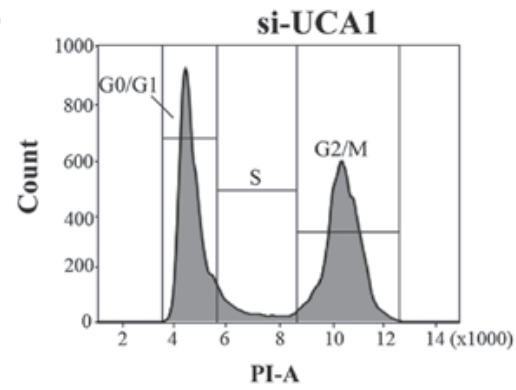

D
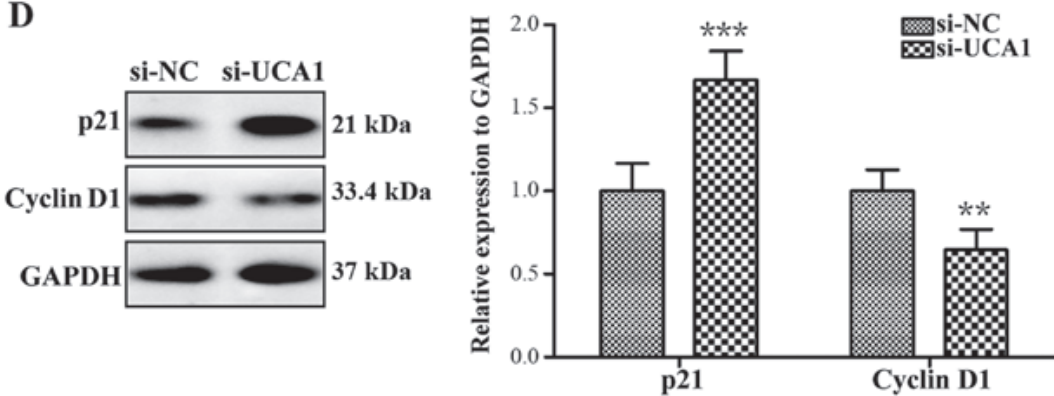

Figure 4. UCA1 silencing promotes $\mathrm{G}_{2} / \mathrm{M}$ phase cell cycle arrest following tamoxifen treatment. (A-C) si-NC LCC2 and si-UCA1 LCC2 cells treated with $10 \mu \mathrm{M}$ tamoxifen exhibited increased $\mathrm{G}_{2} / \mathrm{M}$ cell cycle distribution. (D) Western blot analysis determined the expression levels of p21 and cyclin D1 in si-NC LCC2 and si-UCA1 LCC2 cells. GAPDH was used as a loading control. Data were analyzed using the independent samples Student's t-test. ${ }^{* *} \mathrm{P}<0.01$ and

${ }^{* * *} \mathrm{P}<0.001$ vs. control groups. UCA1, urothelial cancer associated 1; si, small interfering RNA; NC, negative control.

Bcl-2, cleaved caspase-3 and cleaved caspase-9 were significantly increased in si-UCA1 LCC2 cells (Fig. 3E and F; $\mathrm{P}<0.05$ and $\mathrm{P}<0.001)$. These data suggest that UCA1 contributed to the tamoxifen resistance in breast cancer cells.

UCA1 silencing promotes $G_{2} / M$ phase cell cycle arrest following tamoxifen treatment. A previous study have demonstrated that UCA1 could promote bladder cancer progression (33). Therefore, the cell cycle distribution in LCC2 cells post-UCA1 knockdown was assessed. si-UCA1 LCC2 cells treated with $10 \mu \mathrm{M}$ tamoxifen for $24 \mathrm{~h}$ exhibited significant $\mathrm{G}_{2} / \mathrm{M}$ phase arrest (Fig. $4 \mathrm{~A}-\mathrm{C} ; \mathrm{P}<0.01$ ), and the expression level of cell cycle-associated factor p21 was significantly upregulated and the expression level of cyclin D1 was significantly downregulated (Fig. 4D; $\mathrm{P}<0.001$ and $\mathrm{P}<0.01$ ).

UCA1 recruits EZH2 to the $p 21$ promoter and represses $p 21$ expression. It was reported that EZH2 could inhibit the expression of p21 and that p21 is a target of UCA1 (41). It was speculated in the present cell model that p21 may also be suppressed by UCA1 through the recruitment of EZH2 on the p21 promoter. Therefore, RIP analysis was performed. The results indicated that, compared with the IgG control antibody, UCA1 was significantly enriched by EZH2 antibody (Fig. 5A; $\mathrm{P}<0.01)$.
ChIP analysis was further performed to demonstrate whether UCA1 inhibited $\mathrm{p} 21$ expression by interacting with EZH2. As indicated in Fig. 5B, EZH2 and H3K27me3 could bind to the p21 promoter region directly. However, in the si-UCA1 LCC 2 cells, the binding of EZH2 and H3K27me 3 to the p21 promoter region was significantly weakened $(\mathrm{P}<0.01)$. This finding suggested that UCA1 repressed the expression of p21 via the recruitment of EZH2 and H3K27me3.

UCA1 contributes to tamoxifen resistance in breast cancer cells through the PI3K/AKT signaling pathway. CREB-binding protein, a key nuclear transcription factor in the PI3K/AKT signaling pathway, serves an important role in cell cycle progression (42). A previous study demonstrated that cell cycle progression was greatly arrested in UCA1 knockdown cells, and CREB expression levels were significantly downregulated simultaneously (33). In the present study, it was investigated whether UCA1 could influence the expression of CREB. As indicated in Fig. 6A, CREB and p-CREB expression levels were reduced in si-UCA1 LCC2 cells. Band density analysis revealed that the level of CREB and the p-CREB expression significantly decreased 3.06-fold and 2.1-fold when compared with the control group (Fig. 6B; $\mathrm{P}<0.001$ and $\mathrm{P}<0.01$ ).

Considering that the PI3K/AKT signaling pathway is pivotal for the maintenance of normal cell cycle progression 

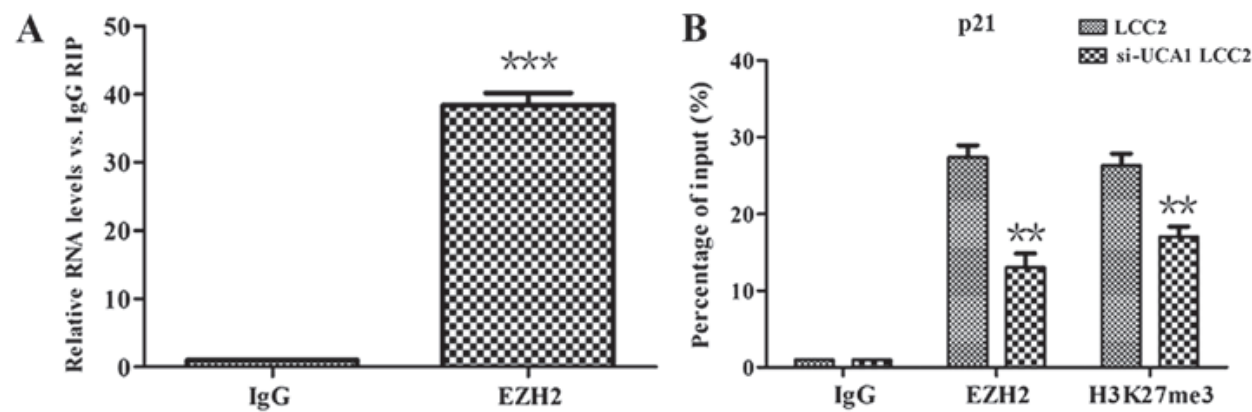

Figure 5. UCA1 represses the expression of p21 via the recruitment of EZH2 in breast cancer cells. (A) UCA1 immunoprecipitated with EZH2 was determined by quantitative polymerase chain reaction and presented as fold enrichment relative to IgG immunoprecipitates in breast cancer cells. (B) Chromatin immunoprecipitation quantitative polymerase chain reaction was used to analyze EZH2 and $\mathrm{H} 3 \mathrm{~K} 27 \mathrm{me} 3$ binding to the p21 promoter regions in LCC2 and si-UCA1 LCC2 cells. IgG was used as a negative control. Data were analyzed using the independent samples Student's t-test. ${ }^{* *} \mathrm{P}<0.01$ and ${ }^{* * * *} \mathrm{P}<0.001$ vs. control group. UCA1, urothelial cancer associated 1; si, small interfering RNA; EZH2, enhancer of zeste homolog 2.

A

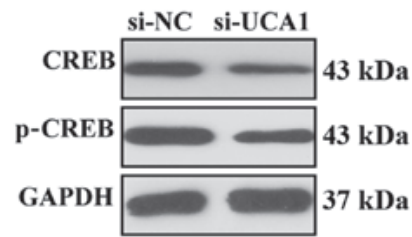

B

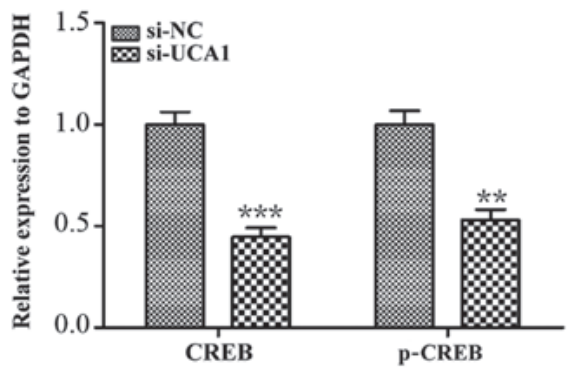

Figure 6. UCA1 silencing reduces CREB and p-CREB expression. (A) Western blot analysis revealed that the expression of CREB and p-CREB was reduced in si-UCA1 LCC2 cells compared with si-NC LCC2 cells. (B) Relative band density of the blots normalized to GAPDH. Data were analyzed using independent samples Student's t-test. ${ }^{* *} \mathrm{P}<0.01$ and ${ }^{* * *} \mathrm{P}<0.001$ vs. control group. UCA1, urothelial cancer associated 1; si, small interfering RNA; NC, negative control; p-, phospho; CREB, CAMP responsive element binding protein.

and is associated with CREB expression $(43,44)$, it was further assessed whether the PI3K/AKT signal pathway could regulate the expression of CREB in si-UCA1 LCC2 cells in the present study. As indicated in Fig. 7, the expression levels of AKT and p-AKT were significantly reduced in si-UCA1 LCC2 cells $(\mathrm{P}<0.0001$ and $\mathrm{P}<0.01)$, suggesting that UCA1 was involved in the activation of AKT.

In order to further verify whether UCA1 could regulate CREB through the PI3K/AKT signaling pathway, LCC2 cells were treated with the PI3K inhibitor LY294002 for 24 h. qPCR analysis revealed that LCC2 cells treated with LY294002 exhibited significantly decreased UCA1 expression levels (Fig. 8A; $\mathrm{P}<0.01$ ). Furthermore, the phosphorylation of CREB and AKT was also significantly repressed in LCC2 cells were treated with LY294002 (Fig. 8B and C; P<0.001 and P<0.05). Taken together, these results further indicated that UCA1 regulated the activation of CREB and impacted cell cycle progression through PI3K/AKT-dependent signaling.
A

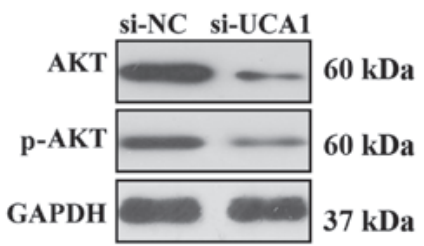

B

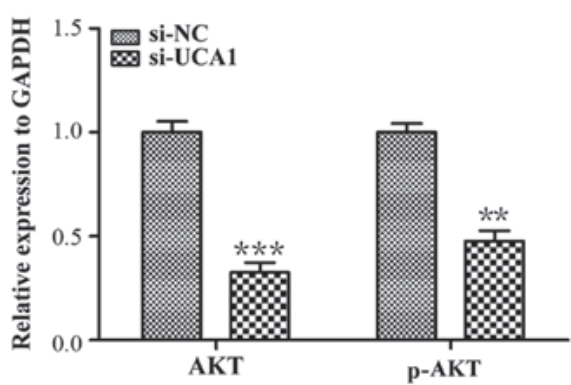

Figure 7. UCA1 silencing represses the activation of AKT. (A) Western blot analysis revealed that the expression of AKT and p-AKT were greatly reduced in si-UCA1 LCC2 cells compared with si-NC LCC2 cells. (B) Relative band density of the blots normalized to GAPDH. Data were analyzed using independent samples Student's t-test. ${ }^{* *} \mathrm{P}<0.01$ and ${ }^{* * *} \mathrm{P}<0.001$ vs. control group. UCA1, urothelial cancer associated 1; si, small interfering RNA; NC, negative control; p-, phospho.

\section{Discussion}

Breast cancer currently remains the most common female malignancy in the world (45). Tamoxifen is the most frequently used endocrinotherapy for $\mathrm{ER}^{+}$breast cancer (46). Despite great treatment advances in improving the survival rate of patients with breast cancer, almost $30 \%$ of patients treated with tamoxifen may develop resistance to the drug (47). Numerous studies have focused on the function of lncRNA, and emerging evidence has demonstrated that lncRNAs significantly contribute to various aspects of cancer biology and have been identified as critical players of drug resistance in cancer therapy (44). However, the underlying mechanisms for tamoxifen resistance are largely unknown. In the present study, it was indicated that UCA1 expression was significantly increased in tamoxifen-resistant breast cancer compared with tamoxifen-sensitive breast cancer. Following the knockdown of UCA1, breast cancer cells exhibited a significant increase in $\mathrm{G}_{2} / \mathrm{M}$ phase cell cycle arrest. 

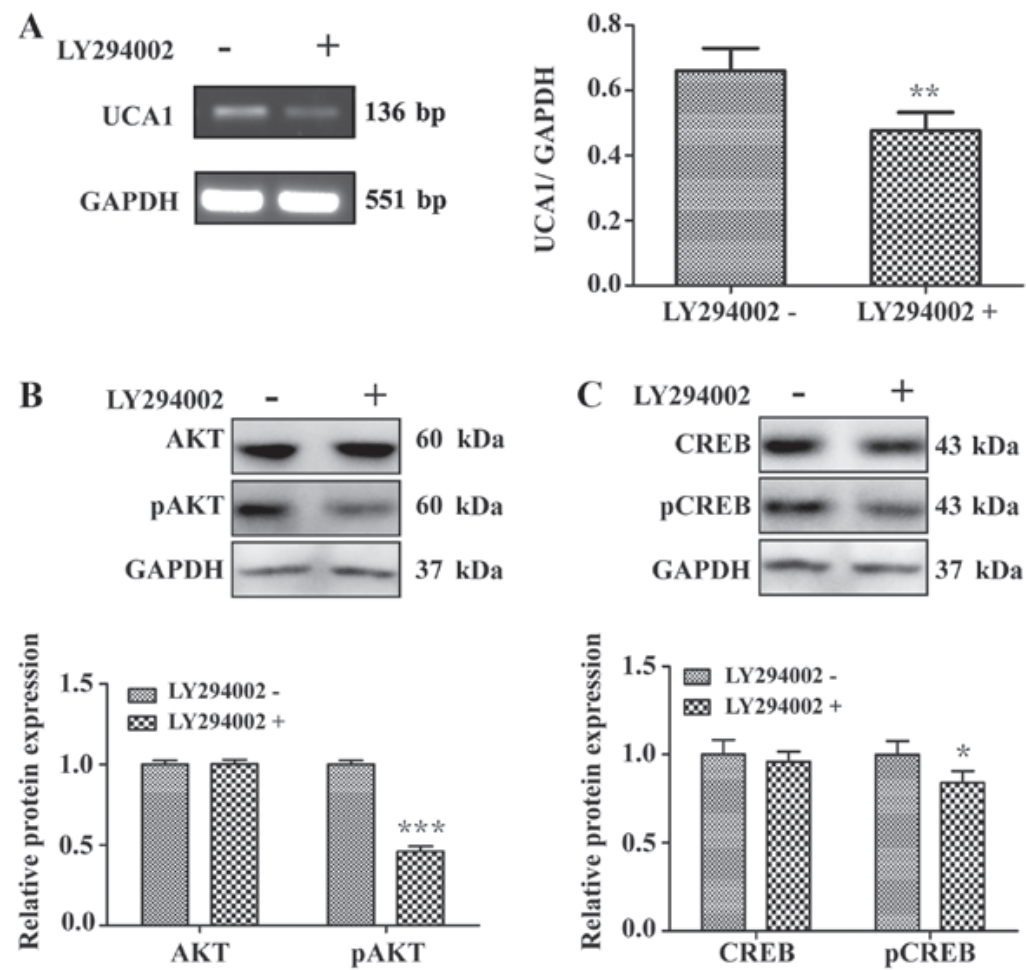

Figure 8. UCA1 regulates the activation of CREB through PI3K/AKT-dependent signaling. (A) Quantitative polymerase chain reaction analysis indicated that the PI3K pathway inhibitor, LY294002, inhibited the expression of UCA1 in LCC2 cells. (B) The expression of AKT/p-AKT was determined by western blot analysis. (C) The expression of CREB/p-CREB was determined by western blot analysis. Data were analyzed using independent samples Student's t-test. ${ }^{*} \mathrm{P}<0.05,{ }^{* *} \mathrm{P}<0.01$ and ${ }^{* * *} \mathrm{P}<0.001$ vs. control groups. CREB, CAMP responsive element binding protein; $\mathrm{p}-$, phospho; UCA1, urothelial cancer associated 1; PI3K, phosphoinositide 3-kinase.

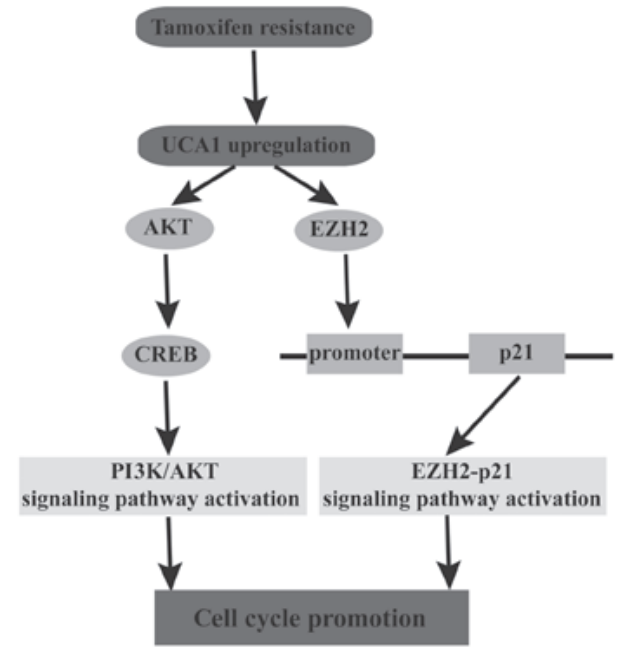

Figure 9. Diagram depicting the role of UCA1 in tamoxifen resistance in breast cancer. UCA1 is physically associated with EZH2, and suppresses the expression of $\mathrm{p} 21$ by impacting the binding of $\mathrm{H} 3 \mathrm{~K} 27 \mathrm{me} 3$ on the $\mathrm{p} 21$ promoter. Furthermore, UCA1 impacts the activation of CREB and AKT, and contributes to tamoxifen resistance via PI3K/AKT-dependent signaling in human breast cancer. UCA1, urothelial cancer associated 1; PI3K, phosphoinositide 3-kinase; EZH2, enhancer of zeste homolog 2.

UCA1 has been reported to be upregulated and to exert its oncogenic activity and enhance chemoresistance in several cancer types $(23,26,35,48)$. It has been reported that UCA1 can increase chemosensitivity through a CREB-miR-196a-5p paradigm in bladder cancer (49). Various studies have demonstrated that UCA1 expression is elevated in breast cancer. For example, Liu et al (50) revealed that UCA1 regulates tamoxifen resistance through the $\mathrm{Wnt} / \beta$-catenin signaling pathway in breast cancer. Consistent with these reports, in the present study it was demonstrated that UCA1 was significantly increased in tamoxifen-resistant breast cancer. Following treatment with tamoxifen, the expression levels of Bcl-2 and cleaved caspase- 3 and -9 were increased in si-UCA1 LCC2 and si-UCA1 LCC9 cells, which demonstrated that UCA1 contributed to tamoxifen drug resistance in breast cancer cells. Bcl-2 protein is a critical component in cell apoptotic signaling. It blocks the increased permeability of the mitochondrial membrane and prevents the release of cytochrome $c$ (51). Several studies have reported lncRNA-mediated sequestering of miR expression, whereas some miRs can directly target $\mathrm{Bcl}-2$ and affect the function of Bcl-2 (52-54). It was presumed that UCA1 regulated Bcl-2 through a similar manner. However, the exact reason for this change remains to be further studied.

The PI3K/AKT signaling pathway serves an important role in cell growth, cell cycle distribution, apoptosis and survival of human cancer (55). AKT and CREB are two key molecules in this pathway. IncRNA may regulate the activation of the PI3K/AKT signaling pathway and affect tumorigenesis and drug sensitivity. For example, miR-21 can modulate tamoxifen sensitivity of breast cancer cells through the PI3K/AKT/mTOR signaling pathway (56). In the present study, it was demonstrated that knockdown of UCA1 in LCC2 cells induced an apparent $\mathrm{G}_{2} / \mathrm{M}$ phase arrest and altered the expression of $\mathrm{p} 21$ and cyclin D1.

A previous study reported that $\mathrm{p} 21$ transcription could be repressed through recruitment of $\mathrm{EZH} 2$, which was mediated 
by UCA1 in renal cell carcinoma cells (40). EZH2 is a histone methyltransferase that catalyzes the trimethylation of $\mathrm{H} 3 \mathrm{~K} 27 \mathrm{me} 3$ of target genes. The levels of EZH 2 are frequently elevated in breast cancer (30). The present study indicated that p21 transcription was repressed by EZH2 through H3K27me3, which was mediated by UCA1 in breast cancer cells. These data demonstrated that UCA1 could modulate the cell cycle through EZH2 and $\mathrm{H} 3 \mathrm{~K} 27 \mathrm{me} 3$ in breast cancer cells.

CREB, a proto-oncogenic transcription factor, is crucial in cell cycle regulation of breast cancer cells (57). In the present study, the association between the expression of UCA1 with the expression of CREB was assessed by western blot analysis. Results demonstrated that CREB and p-CREB expression levels were significantly decreased when UCA1 was suppressed. CREB is mediated by various protein kinases, including AKT and PI3K (58). Likewise, it was indicated in the present study that AKT expression was positively associated with UCA1 expression. A previous study reported that CREB could be positively regulated by AKT kinase activity (33). Furthermore, the present results confirmed that the expression levels of p-AKT and p-CREB were inhibited by the PI3K inhibitor, LY294002, and this was consistent with a previous report (33). These data demonstrated that UCA1 could regulate CREB through AKT via PI3K/AKT signaling.

In conclusion, to the best of our knowledge the present study demonstrated for the first time that UCA1 regulates tamoxifen resistance through the EZH2/p21 axis and the PI3K/AKT signaling pathway in breast cancer (Fig. 9). Based on the present results, UCA1 may be considered a novel biomarker of poor response to tamoxifen and a potential therapeutic intervention target of breast cancer endocrinotherapy.

\section{Acknowledgements}

Not applicable.

\section{Funding}

The present study was supported in part by grants from the Natural Science Foundation of Jilin University (Bethune plan B) (grant no. 2015311 was awarded to ZL).

\section{Availability of data and materials}

The datasets used and analyzed during the current study are available from the corresponding author on reasonable request.

\section{Authors' contributions}

SL contributed to the design of the study and wrote the manuscript. ZL and DY performed the experiments and analyzed the data. HL and YL helped perform the analysis with constructive discussions. All authors have read and approved this manuscript.

\section{Ethics approval and consent to participate}

Permission to use the clinical samples for research purposes was obtained and approved by the Ethics Committee of the First Hospital of Jilin University.

\section{Patient consent for publication}

Informed consents were obtained from all patients.

\section{Competing interests}

The authors declare no conflict of interest.

\section{References}

1. Beiki O, Hall P, Ekbom A and Moradi T: Breast cancer incidence and case fatality among 4.7 million women in relation to social and ethnic background: A population-based cohort study. Breast Cancer Res 14: R5, 2012.

2. Lim E, Metzger-Filho O and Winer EP: The natural history of hormone receptor-positive breast cancer. Oncology (Williston Park) 26: 688-694, 696, 2012

3. Regan MM, Neven P, Giobbie-Hurder A, Goldhirsch A, Ejlertsen B, Mauriac L, Forbes JF, Smith I, Láng I, Wardley A, et al; BIG 1-98 Collaborative Group; International Breast Cancer Study Group (IBCSG): Assessment of letrozole and tamoxifen alone and in sequence for postmenopausal women with steroid hormone receptor-positive breast cancer: The BIG 1-98 randomised clinical trial at $8 \cdot 1$ years median follow-up. Lancet Oncol 12: 1101-1108, 2011.

4. Colditz GA: Relationship between estrogen levels, use of hormone replacement therapy, and breast cancer. J Natl Cancer Inst 90: 814-823, 1998.

5. Lumachi F, Brunello A, Maruzzo M, Basso U and Basso SM: Treatment of estrogen receptor-positive breast cancer. Curr Med Chem 20: 596-604, 2013

6. Higgins MJ and Stearns V: CYP2D6 polymorphisms and tamoxifen metabolism: Clinical relevance. Curr Oncol Rep 12: 7-15, 2010.

7. Raha P, Thomas S and Munster PN: Epigenetic modulation: A novel therapeutic target for overcoming hormonal therapy resistance. Epigenomics 3: 451-470, 2011.

8. Hurvitz SA and Pietras RJ: Rational management of endocrine resistance in breast cancer: A comprehensive review of estrogen receptor biology, treatment options, and future directions. Cancer 113: 2385-2397, 2008.

9. Anzick SL, Kononen J, Walker RL, Azorsa DO, Tanner MM, Guan XY, Sauter G, Kallioniemi OP, Trent JM and Meltzer PS: AIB1, a steroid receptor coactivator amplified in breast and ovarian cancer. Science 277: 965-968, 1997.

10. Ravdin PM, Fritz NF, Tormey DC and Jordan VC: Endocrine status of premenopausal node-positive breast cancer patients following adjuvant chemotherapy and long-term tamoxifen. Cancer Res 48: 1026-1029, 1988.

11. Paridaens R, Sylvester RJ, Ferrazzi E, Legros N, Leclercq G and Heuson JC: Clinical significance of the quantitative assessment of estrogen receptors in advanced breast cancer. Cancer 46 (Suppl): 2889-2895, 1980.

12. Di Gesualdo F, Capaccioli S and Lulli M: A pathophysiological view of the long non-coding RNA world. Oncotarget 5: 10976-10996, 2014.

13. Li Z, Shen J, Chan MT and Wu WK: TUG1: A pivotal oncogenic long non-coding RNA of human cancers. Cell Prolif 49: 471-475, 2016.

14. Han P and Chang CP: Long non-coding RNA and chromatin remodeling. RNA Biol 12: 1094-1098, 2015.

15. Kornienko AE, Guenzl PM, Barlow DP and Pauler FM: Gene regulation by the act of long non-coding RNA transcription. BMC Biol 11: 59, 2013.

16. Zhang HY, Liang F, Zhang JW, Wang F, Wang L and Kang XG: Effects of long noncoding RNA-ROR on tamoxifen resistance of breast cancer cells by regulating microRNA-205. Cancer Chemother Pharmacol 79: 327-337, 2017.

17. Li X, Wu Y, Liu A and Tang X: Long non-coding RNA UCA1 enhances tamoxifena resistance in breast cancer cells through a miR-18a-HIF1 $\alpha$ feedback regulatory loop. Tumor Biol 37: 14733-14743, 2016

18. Cai Y, He J and Zhang D: Suppression of long non-coding RNA CCAT2 improves tamoxifen-resistant breast cancer cells' response to tamoxifen. Mol Biol 50: 821-827, 2016 (In Russian). 
19. Chen G, Wang Z, Wang D, Qiu C, Liu M, Chen X, Zhang Q, Yan G and CuiQ: lncRNADisease: Adatabase for long-non-coding RNA-associated diseases. Nucleic Acids Res 41D: D983-D986, 2013.

20. Lottin S, Adriaenssens E, Dupressoir T, Berteaux N, Montpellier C, Coll J, Dugimont T and Curgy JJ: Overexpression of an ectopic H19 gene enhances the tumorigenic properties of breast cancer cells. Carcinogenesis 23: 1885-1895, 2002.

21. Gupta RA, Shah N, Wang KC, Kim J, Horlings HM, Wong DJ, Tsai MC, Hung T, Argani P, Rinn JL, et al: Long non-coding RNA HOTAIR reprograms chromatin state to promote cancer metastasis. Nature 464: 1071-1076, 2010.

22. Xue M, Chen W and Li X: Urothelial cancer associated 1: A long noncoding RNA with a crucial role in cancer. J Cancer Res Clin Oncol 142: 1407-1419, 2016.

23. Huang J, Zhou N, Watabe K, Lu Z, Wu F, Xu M and Mo YY: Long non-coding RNA UCA1 promotes breast tumor growth by suppression of p27 (Kip1). Cell Death Dis 5: e1008, 2014.

24. Wang XS, Zhang Z, Wang HC, Cai JL, Xu QW, Li MQ, Chen YC, Qian XP, Lu TJ, Yu LZ, et al: Rapid identification of UCA1 as a very sensitive and specific unique marker for human bladder carcinoma. Clin Cancer Res 12: 4851-4858, 2006.

25. Tuo YL, Li XM and Luo J: Long noncoding RNA UCA1 modulates breast cancer cell growth and apoptosis through decreasing tumor suppressive miR-143. Eur Rev Med Pharmacol Sci 19: 3403-3411, 2015.

26. Bian Z, Jin L, Zhang J, Yin Y, Quan C, Hu Y, Feng Y, Liu H, Fei $\mathrm{B}$, Mao Y, et al: lncRNA-UCA1 enhances cell proliferation and 5-fluorouracil resistance in colorectal cancer by inhibiting miR-204-5p. Sci Rep 6: 23892, 2016.

27. Li GY, Wang W, Sun JY, Xin B, Zhang X, Wang T, Zhang QF, Yao LB, Han H, Fan DM, et al: Long non-coding RNAs AC026904.1 and UCA1: A 'one-two punch' for TGF- $\beta$-induced SNAI2 activation and epithelial-mesenchymal transition in breast cancer. Theranostics 8: 2846-2861, 2018.

28. Ringrose L and Paro R: Epigenetic regulation of cellular memory by the Polycomb and Trithorax group proteins. Annu Rev Genet 38: 413-443, 2004

29. Haupt Y, Alexander WS, Barri G, Klinken SP and Adams JM: Novel zinc finger gene implicated as myc collaborator by retrovirally accelerated lymphomagenesis in E mu-myc transgenic mice. Cell 65: 753-763, 1991.

30. Sauvageau M and Sauvageau G: Polycomb group proteins: Multi-faceted regulators of somatic stem cells and cancer. Cell Stem Cell 7: 299-313, 2010

31. Yap DB, Chu J, Berg T, Schapira M, Cheng SW, Moradian A, Morin RD, Mungall AJ, Meissner B, Boyle M, et al: Somatic mutations at EZH2 Y641 act dominantly through a mechanism of selectively altered PRC2 catalytic activity, to increase H3K27 trimethylation. Blood 117: 2451-2459, 2011

32. Hu JJ, Song W, Zhang SD, Shen XH, Qiu XM, Wu HZ, Gong PH, Lu S, Zhao ZJ, He ML, et al: HBx-upregulated lncRNA UCA1 promotes cell growth and tumorigenesis by recruiting EZH2 and repressing p27Kip1/CDK2 signaling. Sci Rep 6: 23521, 2016.

33. Yang C, Li X, Wang Y, Zhao L and Chen W: Long non-coding RNA UCA1 regulated cell cycle distribution via CREB through PI3-K dependent pathway in bladder carcinoma cells. Gene 496: $8-16,2012$

34. Xie X, Pan J, Wei L, Wu S, Hou H, Li X and Chen W: Gene expression profiling of microRNAs associated with UCA1 in bladder cancer cells. Int J Oncol 48: 1617-1627, 2016.

35. Chen S, Shao C, Xu M, Ji J, Xie Y, Lei Y and Wang X: Macrophage infiltration promotes invasiveness of breast cancer cells via activating long non-coding RNA UCA1. Int J Clin Exp Pathol 8: 9052-9061, 2015.

36. You D, Jung SP, Jeong Y, Bae SY, Lee JE and Kim S: Fibronectin expression is upregulated by PI-3K/Akt activation in tamoxifen-resistant breast cancer cells. BMB Rep 50: 615-620, 2017.

37. Xue M, Li X, Li Z and Chen W: Urothelial carcinoma associated 1 is a hypoxia-inducible factor-1 $\alpha$-targeted long noncoding RNA that enhances hypoxic bladder cancer cell proliferation, migration, and invasion. Tumour Biol 35: 6901-6912, 2014.

38. Motawi TK, Abdelazim SA, Darwish HA, Elbaz EM and Shouman SA: Modulation of tamoxifen cytotoxicity by caffeic acid phenethyl ester in MCF-7 breast cancer cells. Oxid Med Cell Longev 2016: 3017108, 2016.

39. Grigsby JG, Parvathaneni K, Almanza MA, Botello AM, Mondragon AA, Allen DM and Tsin AT: Effects of tamoxifen versus raloxifene on retinal capillary endothelial cell proliferation. J Ocul Pharmacol Ther 27: 225-233, 2011
40. Lu Y, Liu WG, Lu JH, Liu ZJ, Li HB, Liu GJ, She HY, Li GY and Shi XH: lncRNA UCA1 promotes renal cell carcinoma proliferation through epigenetically repressing $\mathrm{p} 21$ expression and negatively regulating miR-495. Tumour Biol 39: $1010428317701632,2017$.

41. Chen WM, Huang MD, Sun DP, Kong R, Xu TP, Xia R, Zhang EB and Shu YQ: Long intergenic non-coding RNA 00152 promotes tumor cell cycle progression by binding to EZH2 and repressing p15 and p21 in gastric cancer. Oncotarget 7: 9773-9787, 2016.

42. Giordano A and Avantaggiati ML: p300 and CBP: Partners for life and death. J Cell Physiol 181: 218-230, 1999.

43. Du J, Tong A, Wang F, Cui Y, Li C, Zhang Y and Yan Z: The Roles of PI3K/AKT/mTOR and MAPK/ERK Signaling Pathways in Human Pheochromocytomas. Int J Endocrinol 2016: 5286972 , 2016.

44. Liang MH, Wendland JR and Chuang DM: Lithium inhibits Smad3/4 transactivation via increased CREB activity induced by enhanced PKA and AKT signaling. Mol Cell Neurosci 37: 440-453, 2008

45. Plantamura I, Cosentino G and Cataldo A: MicroRNAs and DNA-damaging drugs in breast cancer: Strength in numbers. Front Oncol 8: 352, 2018.

46. Rocca A, Maltoni R, Bravaccini S, Donati C and Andreis D: Clinical utility of fulvestrant in the treatment of breast cancer: A report on the emerging clinical evidence. Cancer Manag Res 10: 3083-3099, 2018

47. Xia H and Hui KM: Mechanism of cancer drug resistance and the involvement of noncoding RNAs. Curr Med Chem 21: 3029-3041, 2014

48. Cheng N, Cai W, Ren S, Li X, Wang Q, Pan H, Zhao M, Li J, Zhang Y, Zhao C, et al: Long non-coding RNA UCA1 induces non-T790M acquired resistance to EGFR-TKIs by activating the AKT/mTOR pathway in EGFR-mutant non-small cell lung cancer. Oncotarget 6: 23582-23593, 2015.

49. Pan J, Li X, Wu W, Xue M, Hou H, Zhai W and Chen W: Long non-coding RNA UCA1 promotes cisplatin/gemcitabine resistance through CREB modulating miR-196a-5p in bladder cancer cells. Cancer Lett 382: 64-76, 2016.

50. Liu H, Wang G, Yang L, Qu J, Yang Z and Zhou X: Knockdown of long non-coding RNA UCA1 Increases the Tamoxifen Sensitivity of Breast cancer cells through inhibition of Wnt/ $\beta$-catenin pathway. PLoS One 11: e0168406, 2016.

51. Adams JM and Cory S: The Bcl-2 protein family: Arbiters of cell survival. Science 281: 1322-1326, 1998.

52. Deng J, Deng H, Liu C, Liang Y and Wang S: Long non-coding RNA OIP5-AS1 functions as an oncogene in lung adenocarcinoma through targeting $\mathrm{miR}-448 / \mathrm{Bcl}-2$. Biomed Pharmacother 98: 102-110, 2018.

53. Taylor MA, Sossey-Alaoui K, Thompson CL, Danielpour D and Schiemann WP: TGF- $\beta$ upregulates miR-181a expression to promote breast cancer metastasis. J Clin Invest 123: 150-163, 2013.

54. Srivastava N, Manvati S, Srivastava A, Pal R, Kalaiarasan P, Chattopadhyay S, Gochhait S, Dua R and Bamezai RN: miR-24-2 controls H2AFX expression regardless of gene copy number alteration and induces apoptosis by targeting antiapoptotic gene BCL-2: A potential for therapeutic intervention. Breast Cancer Res 13: R39, 2011.

55. Vanhaesebroeck B, Leevers SJ, Ahmadi K, Timms J, Katso R, Driscoll PC, Woscholski R, Parker PJ and Waterfield MD: Synthesis and function of 3-phosphorylated inositol lipids. Annu Rev Biochem 70: 535-602, 2001.

56. Yu X, Li R, Shi W, Jiang T, Wang Y, Li C and Qu X: Silencing of microRNA-21 confers the sensitivity to tamoxifen and fulvestrant by enhancing autophagic cell death through inhibition of the PI3K-AKT-mTOR pathway in breast cancer cells. Biomed Pharmacother 77: 37-44, 2016.

57. de Groot RP, Ballou LM and Sassone-Corsi P: Positive regulation of the cAMP-responsive activator CREM by the p70 S6 kinase: An alternative route to mitogen-induced gene expression. Cell 79: 81-91, 1994.

58. Linnerth NM, Greenaway JB, Petrik JJ and Moorehead RA: cAMP response element-binding protein is expressed at high levels in human ovarian adenocarcinoma and regulates ovarian tumor cell proliferation. Int J Gynecol Cancer 18: 1248-1257, 2008. 